\title{
Lipopeliosis: fat induced sinusoidal dilatation in transplanted liver mimicking peliosis hepatis
}

\author{
L Ferrell, N Bass, J Roberts, N Ascher
}

\begin{abstract}
A distinct peliosis-like lesion arose in the liver allograft of a 51 year old man. This lesion was caused by necrotic, fat-laden hepatocytes that released fat globules into the sinusoids. These then became strikingly distended with cysts, thus mimicking peliosis hepatitis. It is suggested that this lesion be called lipopeliosis.
\end{abstract}

(F Clin Pathol 1992;45:1109-1110)

Several aetiological factors have been implicated in the clinical lesion comprising dilated, blood-filled sinusoids known as peliosis hepatis. ${ }^{1-3}$ Recently, peliosis-like lesions caused by bacillary angiomatosis in the liver have been described. ${ }^{4}$ We now describe another distinct peliosis-like lesion that occurred in a transplanted, steatotic liver following centrilobular hepatocyte injury and necrosis. This lesion was composed of sinusoids dilated by fat globules. Such a lesion has been seen as a complication of using a fatty liver for transplantation. ${ }^{5}$

\section{Case report}

A 51 year old man with alcoholic cirrhosis received a liver transplantation in February 1992. The donor liver was ABO compatible, and seemed to be only a little fatty on gross examination. No initial biopsy was done at the time of transplantation and the donor's history regarding possible predisposing factors for the fatty liver was unknown. The patient's recovery was complicated by severe cholestatic allograft dysfunction and an episode of acute renal failure immediately following transplantation: aspartate aminotransferase (AST) rose to $406 \mathrm{IU} / 1$ (normal 36-122) and alanine aminotransferase (ALT) to $1002 \mathrm{IU} / 1$ (normal 9-50). Both enzymes returned to normal by the eighth postoperative day. Bilirubin rose to $50 \mathrm{mg} / \mathrm{dl}$ (normal $0 \cdot 1-1 \cdot 2$ ) by day 10 , but gradually decreased to $8.6 \mathrm{mg} / \mathrm{dl}$ by day 41 . Alkaline phosphatase activities remained near normal for the first three weeks, but gradually rose over time to three times the normal concentration by day 41 . Liver biopsy specimens were examined at four, 10, 18, 25 and 33 days after surgery. The patient had no evidence of rejection, hepatitis, opportunistic infection, or biliary obstruction or leak following transplantation.

\section{Results}

A biopsy specimen taken six days after transplantation showed large spaces in the centrilobular zone associated with hepatocyte ballooning and necrosis caused by preservation (ischaemic) injury. ${ }^{67}$ These spaces mimicked the cystic, blood-filled spaces seen in peliosis hepatis (fig 1A). Moderate fatty change of both macrovesicular and small droplet types was noted in centrilobular hepatocytes, but only a few foci of small fat droplets were present in periportal zones (fig 1A). Routine haematoxylin and eosin staining showed occasional flattened cells lining the cystic spaces; factor VIII and collagen type IV immunoperoxidase stains were positive along the lining of the spaces, confirming that they were sinusoids (fig 1B). No blood was present in the spaces. Scattered neutrophils and mononuclear cells were also present in the centrilobular zone, and were associated with the necrosis and fat globules. The spaces were still present in the next two biopsy specimens taken 10 and 18 days after surgery, and a frozen section sample stained for neutral lipid by the oil red $O$ technique showed fat globules within them (fig 1C). Two more biopsy specimens taken 25 and 33 days after transplantation showed that the spaces were noticeably smaller, and Kupffer cells with foamy change were the most preva-

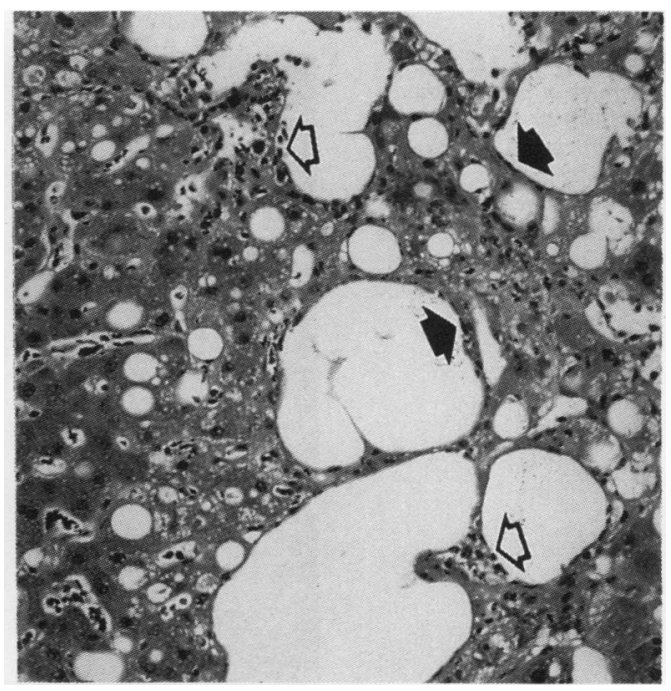

Figure $1 A$ Liver biopsy specimen six days after surgery, showing dilated sinusoidal spaces without blood, mild macro- and microvesicular fatty change in adjacent hepatocytes, and a few scattered inflammatory cells (open arrows). Note the few flat cells lining the spaces that may represent endothelial cells (closed arrows) (haematoxylin and eosin). \\ Pathology, School of Medicine, University of California, San USA \\ Departme
Medicine
N Bass \\ Department of Surgery}


Figure $1 B$ Factor VIII immunoperoxidase stain of the liver at day 6, showing some staining around the large spaces and similar intensity of staining in the non-dilated sinusoids. The space in the centre stains more prominently and represents the central vein (CV).

Figure $1 C$ Oil red $O$ stain of the liver biopsy specimen at day 18 still shows the presence of the dilated sinusoids, containing neutral lipid as shown by the large dark droplets.
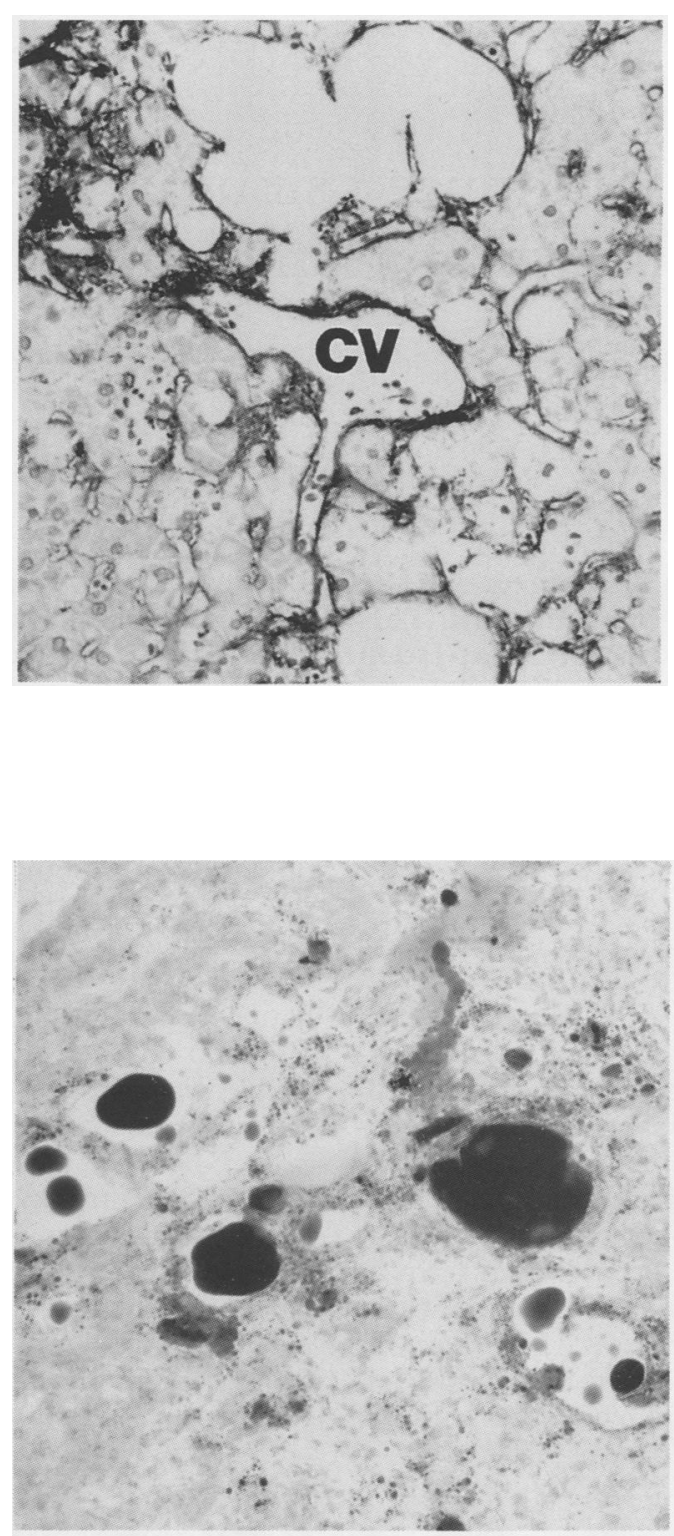

Figure 1D Liver biopsy specimen at day 33 shows centrilobular fibrosis

(arrow) and a decrease in the size of the fatty spaces. Insert: Kupffer cells in the sinusoids contain vacuoles consistent with fat uptake (haematoxylin and eosin).

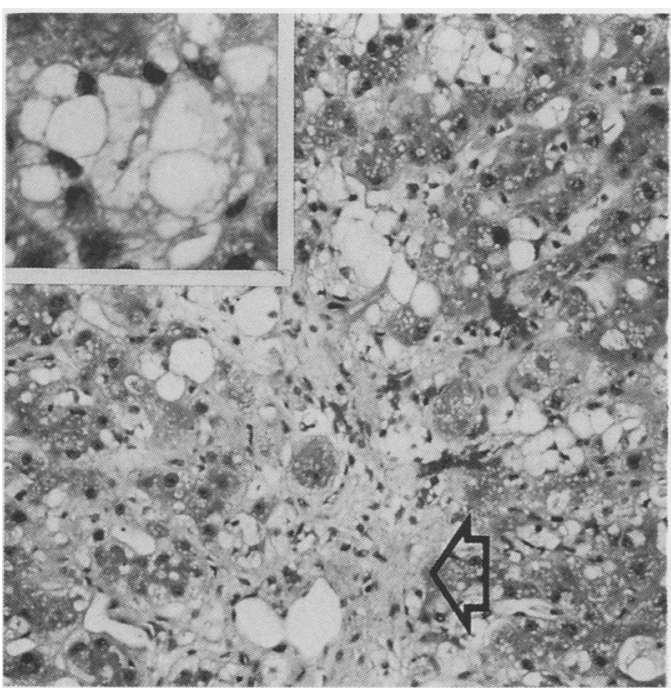

lent inflammatory cell in the sinusoids, especially in the centrilobular zones (fig 1D). The centrilobular zone now showed the presence of fibrosis (fig 1D). Portal zones adjacent to the more severely fibrotic central zones also had foci of mild fibrosis, bile ductular proliferation, and periductular neutrophilic infiltrates, as have been described before, as a consequence of preservation or ischaemic injury. ${ }^{67}$

\section{Discussion}

This case shows a unique lesion associated with steatosis and preservation injury (ischaemic necrosis of the centrilobular hepatocytes) in a hepatic allograft. The mechanism producing this lesion is presumed to be the release of fat from the dying hepatocytes. The fat, in turn, accumulates in, and dilates, the sinusoids, resulting in a histological lesion that superficially resembles peliosis hepatitis. We propose that the term lipopeliosis should be used to describe this distinctive lesion.

A previous report of peliosis hepatis after liver transplantation described the typical lesion of dilated, blood-filled sinusoids in a patient in whom ischaemic liver damage caused by an $\mathrm{ABO}$ incompatible graft might have contributed. ${ }^{3}$ The lesion observed in our patient differs from typical peliosis in the absence of blood in the cystically dilated sinusoids and the striking staining of fat in the spaces. Large extracellular fat globules in dysfunctional hepatic allografts with pre-existing steatosis have been noted in one previous report. $^{5}$ This clearly represents part of the spectrum of the lesion seen in our patient. The pronounced sinusoidal dilation and obstruction by lipopeliosis in the transplanted liver may account, in part, for the frequently severe dysfunction of donor livers which contain a heavy fat infiltrate before transplantation. ${ }^{5}$

Although lipopeliosis may clearly arise in the setting of necrosis of fatty hepatocytes in the donor liver following liver transplantation, it is conceivable that any fatty condition of the liver with a superimposed ischaemic event could result in a similar lesion. We are, however, unaware of any such descriptions.

We thank David Geller for his editorial advice.

1 Simon D, Krause R, Galambos J. Peliosis hepatis in a patient with marasmus. Gastroenterology 1988;95:805-9.

2 Bagheri S, Boyer J. Peliosis hepatis associated with androgenic anabolic

3 Scheur P, Schachter L, Mathur S. Peliosis hepatis after liver transplantation. $f$ Clin Pathol 1990;43:1036-7.

4 Perkocha L, Geaghan S, Yen T, et al. Clinical and pathological features of bacillary peliosis hepatis in association logical features of bacillary peliosis hepatis in association
with HIV infection. $N$ Engl $\mathcal{F}$ Med 1990;323:1581-6.

5 Todo S, Demetris AJ, Makowka L, et al. Primary nonunction of hepatic allografts with pre-existing fatty infiltration. Transplantation 1989;47:903-5.

6 Ray R, Lewin K, Colonna J, Goldstein L, Busuttil R. The role of liver biopsy in evaluating acute allograft dysfunction following liver transplantation. Hum Pathol 1988;19:835-48.

7 Tillery W, Demetris J, Watkins D, et al. Pathologic recognition of preservation injury in hepatis allografts with six months follow up. Transplant Proc 1989;21:1330-1. 